\title{
RESTRICTIONS IMPOSED UPON THE TRANSIENT AND FREQUENCY RESPONSE OF NETWORKS*
}

\author{
BY \\ PAUL M. CHIRLIAN \\ Stevens Institute of Technology
}

Abstract. Readily evaluated upper and lower bounds on the impulse, step, and frequency response of certain classes of networks are obtained. These bounds are the best possible and can be applied to integrated RC structures as well as lumped networks. These procedures can be extended to general networks.

Introduction. One important problem in network analysis is the simple determination of the transient response from the frequency response and, conversely, the determination of the frequency response from the transient response. Estimates or bounds are useful here since they supply considerable information without requiring tedious calculations. In addition, bounds supply theoretical insight into relations between the time and frequency domains.

In this paper we shall determine some such bounds for certain classes of networks. The reader is referred to reference [1] for a discussion of some other bounds.

We shall work with the real part of the frequency response through this paper and write the transient response in terms of it.

Conversely, these bounds can be used to restrict $R(\omega)$. The bounds will be expressed in terms of easily obtained graphical limits and a catalogue of asymptotic responses. Figure 1 is an illustration of the parameters that will be used to bound $R(\omega)$, the real

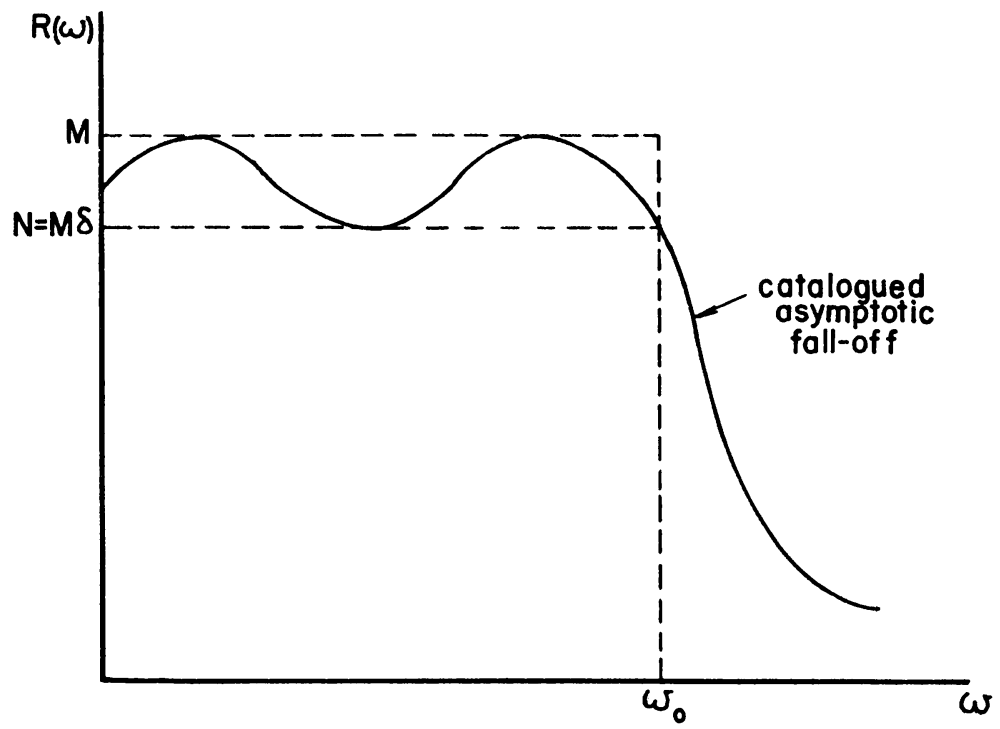

Fig. 1. An illustration of the parameters used to bound $R(\omega)$

${ }^{*}$ Received May 5, 1966; revised manuscript received June 27, 1967. 
part of the system function. That is we shall consider low-pass structures such that

$$
\delta M=N \leq R(\omega) \leq M \text { for } 0 \leq \omega \leq \omega_{0}
$$

and assume that $R(\omega)$ is characterized by a typical asymptotic fall off of either an integrated RC network or a lumped network for $\omega>\omega_{0}$.

We shall use the Fourier transform to obtain bounds on the unit impulse and unit step responses respectively. We shall break each of these integrals into two parts and consider the contributions of the frequencies $0 \leq \omega \leq \omega_{0}$ and $\omega>\omega_{0}$ separately. That is, we write

$$
w(t)=w_{1}(t)+w_{2}(t)=\frac{2}{\pi} \int_{0}^{\omega_{0}} R(\omega) \cos \omega t d \omega+\frac{2}{\pi} \int_{\omega_{0}}^{\infty} R(\omega) \cos \omega t d \omega
$$

and

$$
a(t)=a_{1}(t)+a_{2}(t)=\frac{2}{\pi} \int_{0}^{\omega_{0}} \frac{R(\omega) \sin \omega t}{\omega} d \omega+\frac{2}{\pi} \int_{\omega_{0}}^{\infty} \frac{R(\omega) \sin \omega t}{\omega} d \omega .
$$

Each of these integrals will be considered in turn.

Contributions due to the frequencies $0 \leq \omega \leq \omega_{0}$. Let us consider $w_{1}(t)$ and place bounds upon it. Let $x=\omega t$. Then,

$$
w_{1}(t)=\frac{2}{\pi t} \int_{0}^{\omega_{0} t} R(x / t) \cos x d x .
$$

If $0 \leq \omega_{0} t \leq \pi / 2$, the integral will be maximized if $R(x / t)=M$ and it will be minimized if $R(x / t)=N$. We proceed similarly for greater values of time. That is, to maximize the integral, set $R(x / t)=M$ for those values of $x$ for which $\cos x \geq 0$ and $\operatorname{set} R(x / t)=N$ when $\cos x<0$. Thus, we obtain the following upper bounds

$w_{1}(t) \leq(2 M / \pi t) \sin \omega_{0} t \quad$ for $0 \leq \omega_{0} t \leq \pi / 2$

$w_{1}(t) \leq(2 M / \pi t)\left[k(1-\delta)+\alpha \sin \omega_{0} t\right] \quad$ for $\quad(2 k-1)(\pi / 2) \leq \omega_{0} t<(2 k \mathbf{j}+1) \pi / 2$

$$
k=1,2,3, \cdots
$$

where

$$
\begin{array}{ll}
\alpha=1 & \text { if } k \text { is even, } \\
\alpha=\delta & \text { if } k \text { is odd. }
\end{array}
$$

The lower bounds are given by

$w_{1}(t) \geq(2 M / \pi t) \delta \sin \omega_{0} t \quad$ for $\quad 0 \leq \omega_{0} t \leq \pi / 2$

$w_{1}(t) \geq(2 M / \pi t)\left[k(\delta-1)+(\delta / \alpha) \sin \omega_{0} t\right]$ for $(2 k-1) \pi / 2 \leq \omega_{0} t \leq\left(2 k_{\Delta}+1\right) \pi / 2$

where $\alpha$ is defined in (7).

The previous results were derived on the basis of an infinite number of maxima in $R(\omega)$. If the number of such maxima is limited, then we can improve the bounds.

We must consider the fact that the first region where $\cos x \geq 0$ is only one quarter cycle. Since the number of maxima of $R(\omega)$ is limited we should choose $R(\omega)=M$ for those values of $x$ where $\cos x>0$ for a half cycle. However, at first, we shall restrict ourselves to the case where $R(\omega)$ has its first maximum at $\omega=0$. Thus

$$
w_{1}(t) \leq(2 M / \pi t)\left[(2 \mu-1)(1-\delta)+\delta \sin \omega_{0} t\right] \quad \text { for } \omega_{0} t>-3 \pi / 2+2 \pi \mu .
$$


If we do not restrict the first maximum of $R(\omega)$ to the origin, then we must increase this bound by

$$
\frac{2}{\pi t} \int_{0}^{\pi / 2} M \cos x d x=\frac{2 M}{\pi t} .
$$

The lower bound on $w_{1}(t)$ when the number of maxima of $R(\omega)$ is limited is given by

$$
w_{1}(t) \geq(2 M / \pi t)\left[2 \mu(\delta-1)+\delta \sin \omega_{0} t\right] \quad \text { for } \omega_{0} t>-\pi / 2+2 \pi \mu .
$$

Now let us consider the unit step response. The analysis essentially follows that of the unit impulse response, Hence, we obtain

$$
\begin{aligned}
& a_{1}(t) \leq \frac{2 M}{\pi}\left[(1-\delta) \sum_{0=0}^{k}(-1)^{\nu+1} \mathrm{Si}(v \pi)+\alpha \operatorname{Si}\left(\omega_{0} t\right)\right] \quad \text { for } \quad k \pi \leq \omega_{0} t \leq(k+1) \pi \text {, } \\
& k=0,1,2, \cdots
\end{aligned}
$$

where $\alpha$ is defined in $(7)$ and $\mathrm{Si}\left(\omega_{0} t\right)$ is defined by

$$
\text { Si }(x)=\int_{0}^{x} \frac{\sin y}{y} d y \text {. }
$$

This function is tabulated [2]. The lower bound is given by

$$
\begin{array}{r}
a_{1}(t) \geq \frac{2 M}{\pi}\left[(\delta-1) \sum_{0=0}^{k}(-1)^{\nu+1} \operatorname{Si~}(v \pi)+\frac{\delta}{\alpha} \operatorname{Si~}\left(\omega_{0} t\right)\right] \quad \text { for } k \pi \leq \omega_{0} t<(k+1) \pi, \\
k=0,1,2, \cdots
\end{array}
$$

We now assume that there are only $\mu$ maxima in $R(\omega)$ for $0 \leq \omega \leq \omega_{0}$. Then if $\omega_{0} t \leq(2 \mu-1) \pi$ the results of (13) apply. For $\omega_{0} t>(2 \mu-1) \pi$ we have

$$
a_{1}(t) \leq \frac{2 M}{\pi}\left[(1-\delta) \sum_{v=0}^{2 \mu-1}(-1)^{v+1} \mathrm{Si}(v \pi)+\delta \mathrm{Si}\left(\omega_{0} t\right)\right] .
$$

The results of (14) apply for the lower bound if $\omega_{0} t \leq 2 \pi \mu$. For $\omega_{0} t>2 \pi \mu$, we have

$$
a_{1}(t) \geq \frac{2 M}{\pi}\left[(\delta-1) \sum_{\nu=0}^{2 \mu}(-1)^{\nu+1} \operatorname{Si}(v \pi)+\delta \operatorname{Si}\left(\omega_{0} t\right)\right] .
$$

Thus bounds have been imposed upon $w_{1}(t)$ and $a_{1}(t)$. These are the best possible bounds since for any value of $t$ an $R(\omega)$ can be found that will make $w_{1}(t)$ or $a_{1}(t)$ equal to its bound.

Contributions due to the frequencies $\omega>\omega_{0}$. Now let us consider $w_{2}(t)$ and $a_{2}(t)$. In this case, we shall assume that $R(\omega)$ actually equals one of a catalogue of asymptotic responses. In the subsequent section we shall discuss the error that results when the actual asymptote is not the same as the catalogued ones and discuss a technique for minimizing the error.

One type of fall off that is commonly encountered is

$$
R(\omega)=K\left(\omega_{0} / \omega\right)^{n / 2} .
$$

Substituting and making a change of variable, we obtain

$$
w_{2}(t)=\frac{2 \omega_{0} K}{\pi}\left(w_{0} t\right)^{(n / 2-1)} \int_{\omega_{0} t}^{\infty} \frac{\cos y}{y^{n / 2}} d y .
$$


The integral cannot be evaluated directly. However, tabulated results can be used. If $n=1$, the tables of Fresnel integral [2] are applicable. If $n=2$, we make use of the tabulated integral [2]

$$
\operatorname{ci}(z)=-\int_{z}^{\infty} \frac{\cos x}{x} d x .
$$

For values of $n>2$, the integral of Eq. (18) can be reduced to tabulated forms by successive application of the following integrals [3]:

$$
\int \frac{\cos x}{x^{\rho}} d x=\frac{-1}{\rho-1} \frac{\cos x}{x^{\rho-1}}-\frac{1}{\rho-1} \int \frac{\sin x}{x^{\rho-1}} d x, \quad \rho>1
$$

and

$$
\int \frac{\sin x}{x^{\rho}} d x=\frac{-1}{\rho-1} \frac{\sin x}{x^{\rho-1}}+\frac{1}{\rho-1} \int \frac{\cos x}{x^{\rho-1}} d x, \quad \rho>1 .
$$

Another type of high frequency asymptotic response that occurs quite commonly in integrated networks is the exponential fall off.

$$
R(\omega)=(K n / \cos n) \exp \left[-n\left(\omega / \omega_{0}\right)^{1 / 2}\right] \cos n\left(\omega / \omega_{0}\right)^{1 / 2} .
$$

Substituting and manipulating, we have

$$
w_{2}(t)=\frac{4 \omega_{n} K n}{\pi n^{2} \cos n} \int_{n}^{\infty} x e^{-x} \cos x \cos \frac{\omega_{0} t x^{2}}{n^{2}} d x .
$$

These integrals are not tabulated in the literature. However, they have been evaluated using a computer and are given in Table $\mathrm{I}$.

Now let us consider some values for $a_{2}(t)$. We shall use the same asymptotic responses as before. Then, if $R(\omega)$ is given by (17), we obtain

$$
a_{2}(t)=\frac{2 K}{\pi}\left(\omega_{0} t\right)^{n / 2} \int_{\omega_{0} t}^{\infty} \frac{\sin x}{n^{(n / 2} \frac{x}{11}} d x
$$

This can be reduced to tabulated forms by the successive application of (20) and (21).

In the case of the exponential fall off where $R(\omega)$ is given by (22)

$$
a_{2}(t)=\frac{4}{\pi} \frac{K e^{n}}{\cos n} \int_{n}^{\infty} \frac{e^{-x} \cos x \sin \left[\left(\omega_{0} t x^{2}\right) / n^{2}\right]}{x} d x .
$$

Again, this integral is not tabulated in the literature but it has been evaluated by a computer. The value of

$$
\frac{e^{n}}{\cos n} \int_{n}^{\infty} \frac{e^{-x} \cos x \sin b x^{2}}{x} d x
$$

is given in Table II. Again note that the magnitude of this value falls off as $n$ increases.

Calculation of the bounds on $R(\omega)$. The value of $R(\omega)$ is a function of $w(t)$ and is given by

$$
R(\omega)=R_{1}(\omega)+R_{2}(\omega)=\int_{0}^{t_{0}} w(t) \cos \omega t d t+\int_{t_{0}}^{\infty} w(t) \cos \omega t d t
$$

Thus the previous procedures can be used to bound $R(\omega)$. Logical bounds on $w(t)$ for 
TABLE I

$$
\frac{e^{n}}{n^{2} \cos n} \int_{n}^{\infty} x e^{-x} \cos x \cos b x^{2} d x
$$

\begin{tabular}{|c|c|c|c|c|}
\hline \multirow[b]{2}{*}{$b$} & \multirow[b]{2}{*}{1} & \multicolumn{2}{|c|}{$n$} & \multirow[b]{2}{*}{4} \\
\hline & & 2 & 3 & \\
\hline 0.01 & -1.058 & 1.070 & 0.157 & -0.110 \\
\hline 0.02 & -1.05 & 0.955 & -0.162 & 0.080 \\
\hline 0.03 & -1.058 & 0.555 & -0.186 & -0.046 \\
\hline 0.04 & -1.011 & 0.012 & -0.054 & 0.018 \\
\hline 0.05 & -1.051 & -0.020 & 0.064 & 0.004 \\
\hline 0.06 & -1.032 & -0.039 & 0.102 & 0.019 \\
\hline 0.07 & -0.990 & -0.484 & 0.067 & 0.026 \\
\hline 0.08 & -0.922 & -0.516 & 0.001 & -0.024 \\
\hline 0.09 & -0.831 & -0.506 & -0.053 & 0.016 \\
\hline 0.10 & -0.721 & -0.471 & -0.062 & -0.006 \\
\hline 0.20 & -0.394 & 0.032 & 0.012 & -0.007 \\
\hline 0.30 & 0.765 & 0.119 & 0.016 & -0.006 \\
\hline 0.40 & 0.747 & -0.021 & -0.013 & -0.005 \\
\hline 0.50 & 0.612 & -0.065 & -0.005 & -0.003 \\
\hline 0.60 & 0.459 & 0.015 & 0.010 & -0.002 \\
\hline 0.70 & 0.317 & 0.044 & -0.011 & 0.000 \\
\hline 0.80 & 0.195 & -0.013 & -0.007 & -0.004 \\
\hline 0.90 & 0.095 & -0.034 & 0.004 & 0.013 \\
\hline 1.00 & 0.121 & 0.011 & 0.004 & -0.009 \\
\hline 2.00 & -0.226 & -0.009 & 0.004 & -0.013 \\
\hline 3.00 & -0.082 & 0.007 & 0.352 & -0.001 \\
\hline 4.00 & -0.063 & 0.002 & -0.004 & 0.005 \\
\hline 5.00 & 0.098 & -0.002 & 0.034 & 0.016 \\
\hline 6.00 & 0.039 & -0.025 & 0.084 & -0.004 \\
\hline 7.00 & -0.036 & 0.043 & -0.011 & 0.009 \\
\hline 8.00 & -0.062 & -0.026 & 0.006 & 0.016 \\
\hline 9.00 & -0.030 & -0.060 & 0.031 & 0.023 \\
\hline 10.00 & 0.021 & 0.062 & 0.023 & 0.009 \\
\hline 20.00 & -0.026 & -0.048 & 0.032 & 0.015 \\
\hline 30.00 & -0.044 & -0.012 & -0.028 & -0.025 \\
\hline 40.00 & -0.036 & 0.061 & 0.014 & 0.011 \\
\hline 50.00 & 0.013 & -0.054 & -0.005 & -0.030 \\
\hline 60.00 & 0.107 & -0.135 & 0.030 & -0.000 \\
\hline 70.00 & 0.160 & -0.075 & 0.011 & -0.009 \\
\hline 80.00 & -0.090 & -0.074 & 0.013 & 0.005 \\
\hline 90.00 & -0.003 & 0.195 & -0.183 & 0.019 \\
\hline 100.00 & -0.034 & -0.055 & -0.012 & 0.029 \\
\hline
\end{tabular}

$t \geq t_{0}$ will be exponential decays. Thus, we shall add these responses to our catalogue. If

$$
w(t)=K e^{-t / t_{0}} \text { for } t>t_{0}
$$

then

$$
R_{2}(\omega)=\left(K t_{0} / e\left(1+\omega^{2} t_{0}^{2}\right)\right)\left[\omega t_{1} \sin \omega t_{1}-\cos \omega t_{0}\right]
$$


TABLE II

$$
\frac{e^{n}}{\cos n} \int_{n}^{\infty} \frac{e^{-x} \cos x \sin b x^{2}}{x} d x
$$

\begin{tabular}{|c|c|c|c|c|}
\hline$b$ & 1 & 2 & 3 & 4 \\
\hline 0.01 & -0.011 & 0.171 & 0.163 & 0.053 \\
\hline 0.02 & -0.021 & 0.337 & 0.150 & -0.039 \\
\hline 0.03 & -0.032 & 0.460 & -0.009 & 0.043 \\
\hline 0.04 & -0.042 & 0.513 & -0.109 & -0.043 \\
\hline 0.05 & -0.053 & 0.505 & -0.101 & 0.037 \\
\hline 0.06 & -0.063 & 0.457 & -0.028 & -0.024 \\
\hline 0.07 & -0.073 & 0.386 & 0.044 & 0.009 \\
\hline 0.08 & -0.083 & 0.306 & 0.073 & 0.005 \\
\hline 0.09 & -0.092 & 0.224 & 0.051 & -0.015 \\
\hline 0.10 & -0.100 & 0.146 & 0.002 & 0.019 \\
\hline 0.20 & -0.111 & -0.175 & -0.029 & 0.007 \\
\hline 0.30 & -0.048 & 0.001 & 0.012 & 0.002 \\
\hline 0.40 & 0.029 & 0.084 & 0.009 & -0.001 \\
\hline 0.50 & 0.098 & -0.009 & -0.011 & -0.003 \\
\hline 0.60 & 0.151 & -0.054 & -0.001 & -0.003 \\
\hline 0.70 & 0.190 & 0.009 & 0.009 & -0.003 \\
\hline 0.80 & 0.215 & 0.039 & -0.003 & -0.003 \\
\hline 0.90 & 0.230 & -0.009 & -0.006 & 0.001 \\
\hline 1.00 & 0.235 & -0.030 & 0.005 & 0.001 \\
\hline 2.00 & 0.064 & 0.013 & 0.001 & 0.015 \\
\hline 3.00 & -0.099 & -0.007 & 0.005 & 0.006 \\
\hline 4.00 & -0.101 & 0.002 & 0.003 & 0.008 \\
\hline 5.00 & -0.011 & -0.002 & 0.011 & 0.011 \\
\hline 6.00 & 0.063 & -0.014 & 0.010 & -0.011 \\
\hline 7.00 & 0.062 & -0.016 & 0.004 & -0.005 \\
\hline 8.00 & 0.007 & 0.038 & 0.008 & -0.026 \\
\hline 9.00 & -0.042 & 0.002 & -0.000 & -0.002 \\
\hline 10.00 & -0.045 & -0.042 & -0.006 & 0.020 \\
\hline 20.00 & 0.013 & -0.097 & -0.005 & 0.006 \\
\hline 30.00 & 0.002 & -0.045 & -0.013 & 0.016 \\
\hline 40.00 & -0.016 & -0.027 & -0.011 & 0.012 \\
\hline 50.00 & 0.015 & 0.009 & -0.007 & -0.012 \\
\hline 60.00 & 0.011 & -0.033 & -0.004 & -0.011 \\
\hline 70.00 & 0.008 & -0.020 & 0.033 & -0.016 \\
\hline 80.00 & 0.030 & 0.025 & -0.024 & -0.009 \\
\hline 90.00 & 0.027 & 0.009 & 0.009 & -0.014 \\
\hline 100.00 & -0.025 & -0.003 & -0.040 & 0.001 \\
\hline
\end{tabular}

Accuracy of asymptotic response calculations. In the previous sections, we have assumed that $R(\omega)$ [or $w(t)$ ] was exactly equal to one of a catalogued values of asymptotic responses. In general, they will deviate from these values, which will cause an error in 
$w_{2}(t)$ or $a_{2}(t)$ [or $\left.R(\omega)\right]$. The procedure of [5] can be used to bound the magnitude of this error.

One procedure that can be used to reduce the error is to split the range for $0 \leq \omega \leq \omega_{0}$ into two (or more) ranges each with its own bound on $R(\omega)$. In this way the accuracy of the asymptotic calculations can be increased as desired while there is no sacrifice in the accuracy of $w_{1}(t)$ and $a_{1}(t)$. Often, this not required. Additional values of $R(\omega)$ can be added to the catalogue, also.

Conclusion. A set of readily evaluated bounds on the unit impulse and unit step responses have been obtained. These bounds are best possible since the responses specified by them can actually be obtained. A catalogue of asymptotic responses has been given so that these results can be applied to both integrated and lumped networks.

Acknowledgment. The author wishes to express his thanks to Mr. Bipin Parikh and the staff of the Stevens Institute of Technology computer facility for the evaluation of the integrals of Tables I and II.

\section{ReFERENCES}

[1] A. H. Zemanian, Bounds existing in the time and frequency responses of various types of networks, Proc. IRE 42, 835-839 (1954)

A. H. Zemanian, Further bounds existing on the transient response of various types of networks, Proc. IRE 43, 322-326 (1955)

J. D. Brule, Time-response characteristics of a system as determined by its transfer function, IRE Trans. on Circuit Theory CT-6, 163-170 (1959)

P. M. Chirlian, Bounds on the error in the unit step response of a network, Quart. of Applied Math. 16, 432-435 (1959)

P. M. Chirlian, Restrictions imposed upon the response of linear phase shift networks, Quart. of Applied Math., 17, 225-230 (1959)

P. M. Chirlian, Some necessary conditions for a non-negative unit impulse response and for a positive real immittance function, IRE Trans. on Circuit Theory CT-8, 105-108 (1961)

A. Papoulis, An estimate of the truncation error in Fourier integrals, IRE Trans. on Circuit Theory, CT-9, 186 (1962)

[2] Jahnke-Emde-Lösch, Tables of higher functions, McGraw-Hill, New York, 1960

[3] B. O. Peirce, A short table of integrals, Ginn and Company, Boston, Mass., 1929

[4] W. Grobner and N. Hof reiter, Integraltafel, Springer-Verlag, Vienna, 1957

[5] P. M. Chirlian, Bounds on the error in the unit step response of a network, Quart. of Applied Math. 16, 432-435 (1959)

P. M. Chirlian, Bounds on the error in the unit impulse response of a network, Proc. IRE 48, 262-263 (1960) 